\title{
BIT ERROR RATE ANALYSIS OF DIFFERENT DIGITAL MODULATION SCHEMES IN ORTHOGONAL FREQUENCY DIVISION MULTIPLEXING SYSTEMS
}

\author{
S. O. Ajose ${ }^{1}$, A. L. Imoize ${ }^{2,}{ }^{*}$ and O. M.Obiukwu ${ }^{3}$ \\ 1, 2, 3, DEPARTMENT OF ELECTRICAL \& ELECTRONICS ENGINEERING, UNIVERSITY OF LAGOS, LAGOS STATE, NIGERIA. \\ E-mail addresses: ${ }^{1}$ solumideajose@gmail.com, ${ }^{2}$ aimoize@unilag.edu.ng, ${ }^{3}$ obinnaobiukwu1@gmail.com
}

\begin{abstract}
This study presents the design of an orthogonal frequency division multiplexing (OFDM) system and analyses the performance of the different digital modulation techniques employed in the system. The OFDM system was modelled and different modulation schemes: $M$-ary phase shift keying (M-PSK) and M-ary quadrature amplitude modulation (M-QAM) were employed over two different channels: additive white Gaussian noise (AWGN), and Rayleigh multipath fading channels. Bit error rate (BER) analysis was carried out for the different digital modulation schemes over the two channels, and the number of fast Fourier transform (FFT) points used during the transmission was examined. Generally, results showed that over both AWGN and Rayleigh fading channels, lower order modulation schemes perform better than the higher order schemes. This comes at the detriment of the data rate, as lower order schemes have lower data rates compared with their higher order counterparts. In addition, it was observed that the system performed better over AWGN channel than Rayleigh fading channel for all modulation schemes used. On the number of FFT points used during the transmission, findings revealed that the performance of the system is more or less not really affected by the number of FFT points employed during transmission.
\end{abstract}

Key words: Digital modulation, bit error rate, orthogonal frequency division multiplexing, additive white Gaussian noise, modulation schemes, fast Fourier transform.

\section{INTRODUCTION}

Wireless communication field is ever dynamic and has seen an exponential growth over the past several years [1]. The ever increasing usage of mobile phone technology, Wireless Local Area Network (WLAN) and the rapid growth of the mobile broadband industry have necessitated in finding new means of obtaining higher capacity wireless network [2]. With the increasing traffic for wireless communication, it is inevitable that spectral efficiency and higher data rates would be of paramount importance. This calls for a more bandwidth efficient scheme because spectral efficiency and higher data rates transmission are of utmost significance in wireless communication. Achieving high data rates requires a careful selection of the available multicarrier modulation schemes. Orthogonal frequency division multiplexing (OFDM) is a multi-carrier transmission technique that goes a long way in providing the much-needed improvement as it can be viewed as a multiplexing or modulation technique, which transmits at higher data rates and at the same time improves spectral efficiency [3]. The technique splits up the available spectrum into many subcarriers, and have the transmitter transmit a single data stream over them, each subcarrier being modulated at a lower symbol rate, resulting to a signal with large resistance to interference. This technique is almost identical to frequency division multiple access (FDMA) in that the multiple access is obtained by splitting the available bandwidth into multiple channels, which are now assigned to users. However, OFDM utilises the spectrum more efficiently because the channels are much closer together unlike in FDMA where the channels are being spaced apart with a considerable distance to prevent inter-carrier interference and consequently reducing bit error rates. This eliminates the need for inter carrier guard bands used in FDMA thus simplifying the design of OFDM transceivers.

To prevent the inter-carrier interference in OFDM, the multiple carriers are made orthogonal to each other. Asides the higher data rate transmission and better spectral efficiency, OFDM offers other advantages like robustness against frequency-selective fading channel, efficient implementations using Fast Fourier Transform and simple equalization techniques (that is, low

* Corresponding author, tel: +234-803- $687-3891$ 
computational complexity) [4]. These advantages have developed OFDM into a widely accepted strategy for wideband digital communication transmission used in areas such as digital television and audio broadcasting, wireless networks and $4^{\text {th }}$ generation $(4 \mathrm{G})$ mobile communication [5].

In accordance with the modulation order, the amount of error in the bit information can be varied [6]. Thus, this study concentrated on the analysis of the performance of the OFDM system over different channels whilst employing different modulation techniques. The study was carried out using Simulink in MATLAB and the various digital modulation techniques were discussed in details. These modulation techniques include BPSK, QPSK, 16 QAM, 64 QAM and 256 QAM. The bit error rate (BER) performance of the OFDM system was analysed over both additive white Gaussian noise (AWGN) and Rayleigh fading channels. In addition, the system was analysed over different FFT points to determine if the performance of the system is affected by this factor.

This paper is organized as follows. Section 2 presents previous works on BER analysis of different digital modulation schemes in orthogonal frequency division multiplexing systems, details the OFDM system model and the theoretical background. Simulation implementation of the system is presented in Section 3. In section 4, the simulation results are presented and discussed. Section 5 presents the conclusion to the paper.

\section{RELATED WORKS}

In the existing literature, Kamboj and Kaushik [7] described the basics of the OFDM system and even though a thorough analysis of the modulation schemes was not carried out, emphasis was laid on the constellation analysis of the modulation schemes. Insight was also given on the general study of the OFDM system. The system was also simulated and some performance criteria of the system such as tolerance to multipath delay spread, channel noise and start time error were tested and analysed.

Katariya et al. [8] gave a better insight on the OFDM system and carried out a performance analysis of the modulation schemes under AWGN, basing its modelling and simulation on the IEEE 802.11a standard for WLAN. This standard (IEEE 802.11a) uses 64 FFT points with 52 subcarriers. Three modulation schemes were considered: BPSK, 16QAM and 64 QAM. There were many power gains at higher SNR. However, the analysis in this paper is only over a noisy channel and did not consider multipath channels. Here, it was remarked that the SNR for each modulation takes into account the number of bits per symbol, and so the signal power corresponds to the energy per bit times the number of bits per symbol. It was concluded that the performance of the system will be reduced as the number of constellation mapping points increased from 8 to 64 point, and the higher Eb/No required for transferring data means that more energy is required for each bit transfer.

Instead of using fast Fourier transform to implement orthogonality of the subcarriers, Bodhe et al. [9] used a discrete wave transform (DWT) to do so. This was achieved using MATLAB Simulink. This model, based on DWT was used to analyse the performance of twomodulation scheme: 16QAM and 64QAM. It was found that the former performs better than the latter, though the difference is very marginal. The study also carried out the comparison of FFT based OFDM system, DWT based system employing 64QAM modulation technique, and it was concluded that the performance of the DWTOFDM was far superior to that of FFT-OFDM system. Once again, this study was carried out only over a noisy channel without considering the multipath channels.

Mohamed et al. [10] carried out the performance analysis of the OFDM system by varying some parameters of the system through simulation with MATLAB. It was observed that the more subcarriers used the more accurate and useful the system is over the AWGN channel. Taking an inverse fast Fourier transform (IFFT) size of 1024, it was observed that using M-QAM modulation technique, the optimum value of the SNR is $60 \mathrm{~dB}$ over the AWGN after which there is no improvement in the BER of the system. It was also concluded that the system performed best with the 64QAM.

Dixit Dutt Bohra [11] employed a simulation environment known as LabVIEW to analyse the performance of BPSK and QPSK over the AWGN channel. This system employs low-density parity-check (LPDC) codes, which provides error correction. BPSK was found to have performed better, but once again over the AWGN channel.

Shashikant and Dhawan [12] investigated cyclic prefix (CP) optimization. CP length was varied from $6 \%$ to $100 \%$ of the OFDM useful symbol and it was observed that as CP length exceeds $18.75 \%$, the power loss of the signal becomes more than $1 \mathrm{~dB}$ which reduces the system efficiency. At CP length of $100 \%$, better BER was obtained at a lesser SNR but this is at the expense of $3 \mathrm{~dB}$ signal power loss, thus inefficient. It was suggested that CP length should not exceed $25 \%$ for efficient optimization. 


\subsection{OFDM System Model}

OFDM is a transmission technique based on frequency division multiplexing (FDM). It can be thought of as a multicarrier transmission technique where the subcarriers are orthogonal to each other. Orthogonality is important in this system, as its loss would lead to inter carrier interference (ICI). To maintain the orthogonality, the subcarriers in OFDM are spaced out equally [7].

Typically, the OFDM system has three major parts: the transmitter, channel and receiver as seen in Figure 1.

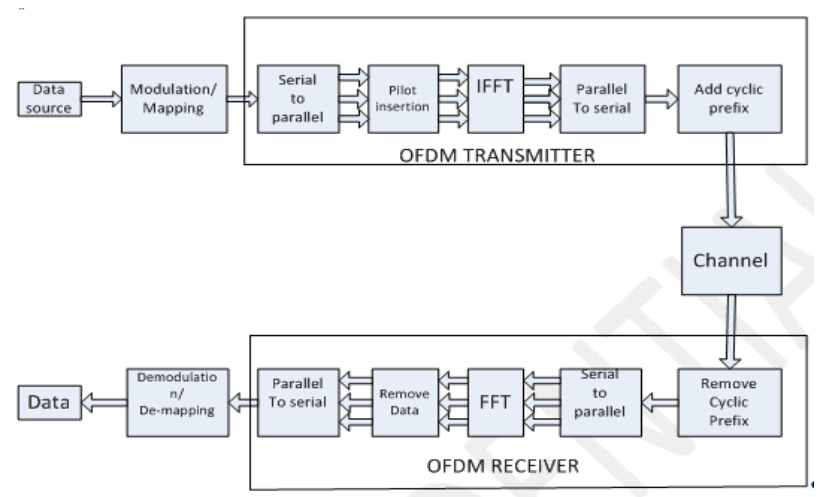

Figure 1: OFDM block diagram

The process starts at the transmitter where incoming data bits are mapped and modulated according to the available modulation scheme. Modulation and mapping are dynamically implemented based on the channel conditions such as Doppler spread and fading in order to improve the performance of the system. The serial bits are converted into parallel stream by the serial to parallel converter. The modulated parallel bit streams undergo baseband OFDM modulation, which is implemented using inverse fast Fourier transform (IFFT). After that, the parallel bits are serialized by the parallel to series converter. Cyclic prefix, whose length is not more than one-fourth of the OFDM symbol duration, is appended to each data symbol in other to prevent inter symbol interference (ISI). The data are now sent to the receiver through the channel. At the receiver, basically, the inverse of the transmitter process occurs. The cyclic prefix is removed, after which the data are converted into a parallel stream by the serial to parallel converter. The parallel data stream are demodulated using fast Fourier transform (FFT) and converted back to a serial stream where they are de-mapped and demodulated to get back the original bits.

\subsection{Mathematical Description of OFDM}

The complex, time domain baseband OFDM signal at the output of the IFFT can be written as [13]:

$$
S_{n}(t)=\frac{1}{N} \sum_{i=0}^{N-1} X_{i} e^{j \frac{2 \pi}{N} n i}
$$

where: $\mathrm{N}$ is the total number of subcarriers,

$X$ is the Amplitude on the subcarrier,

$i$ is the subcarrier index,

$n$ is the time domain sample index of an OFDM signal = $0,1, \ldots \ldots, N-1$.

Equation (1) represents the discrete time OFDM symbol which is the N-point IDFT of PSK or QAM data symbols and can be calculated efficiently by using IFFT.

\subsection{CP Insertion and Removal}

Due to the multipath nature of the transmission radio channel, ISI is introduced to the OFDM signals [14]. Multipath transmission introduces ISI when the maximum delay spread of the radio channel is more than the guard interval length. Hence, the reason why the guard interval length is defined to be more than the maximum delay spread. Thus, the guard interval is added to mitigate the ISI effect [15]. Asides the multipath effect, Doppler shift and carrier instability can cause ISI too, but the guard interval also takes care of those. Generally, the guard interval is introduced to maintain the orthogonality of the subcarriers and the independence of the OFDM symbols, when the OFDM signal is transmitted over a multipath channel.

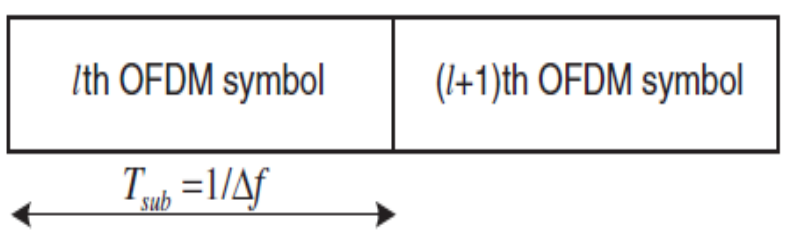

Figure 2: OFDM symbol without guard interval.

The guard interval is introduced through the cyclic extension of the OFDM symbol known as cyclic prefix (CP). The CP is identical to the last samples of the OFDM symbols and is appended at the beginning of the symbol. Let $\mathrm{T}_{\mathrm{G}}$ denote the length of the cyclic prefix in terms of samples. The extended OFDM symbol have the duration of:

$$
T_{\text {sym }}=T_{\text {sub }}+T_{G}
$$

where: $T_{\text {sym }}$ is the total symbol duration, $T_{\text {sub }}$ is the useful symbol duration.

When the cyclic prefix is greater or equals to the maximum delay of a multipath channel, the ISI effect of an OFDM symbol on the next OFDM symbol is restricted to the guard interval so that the effective part of the next OFDM symbol would not be affected with the duration, $\mathrm{T}_{\text {sub }}$. 


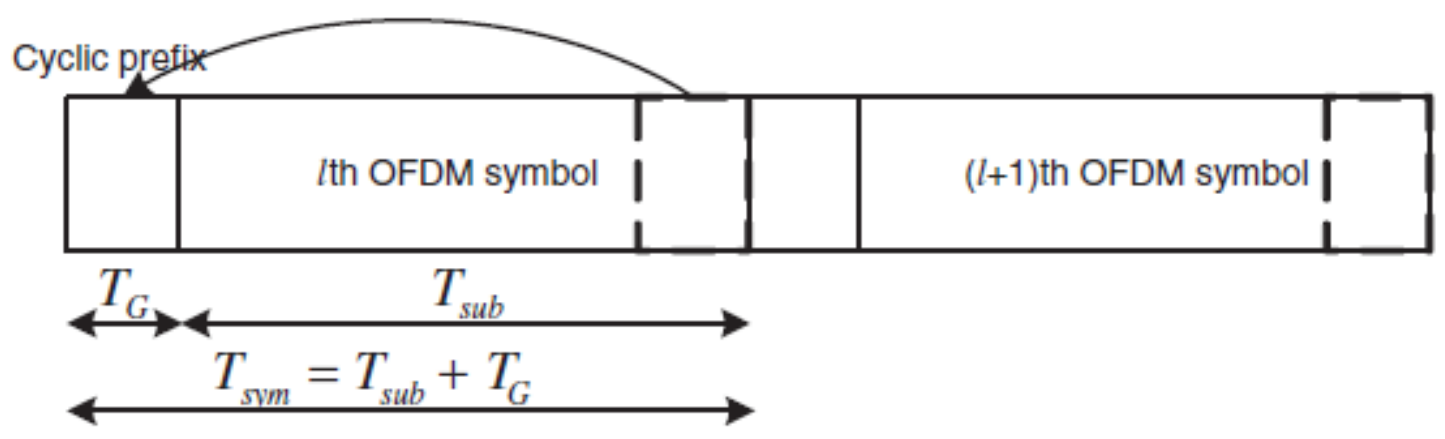

Figure 3: OFDM symbol with cyclic prefix

The consequence of this is that when the guard interval is longer than the maximum delay of the multipath channel, the orthogonality amongst the subcarriers is maintained. Introducing guard interval reduces throughput hence it is usually limited to be less than or equal to one quarter of the useful symbol duration.

\subsection{Digital Modulation in OFDM Systems}

The nature of OFDM only allows the signals to be modulated in amplitude and phase only [4]. This work focuses on PSK and QAM techniques only. The performance of these modulation techniques in a wireless communication system are evaluated in terms of bit error probability.

For binary PSK (BPSK), which is the simplest form of PSK, a pair of signals $S_{1}(t)$ and $S_{2}(t)$ which can be regarded as two phases separated by 180 degrees are used here to represent two binary symbols: 1 and 0 respectively. Typically, these two phases are 0 and 180 degrees. For BPSK, the probability of bit error, $P_{b}$, is given by [16]:

$$
P_{b}=Q \sqrt{\frac{2 E_{b}}{N_{0}}}
$$

where: $E_{b}$ is the Energy per bit, $N_{0}$ noise power spectral density, $Q$ is the error function frequently used for calculating the area under the tail of the Gaussian pdf denoted by $Q_{(x)}$ :

$$
Q_{(x)}=\frac{1}{\sqrt{2 \pi}} \int_{x}^{\infty} e^{-\left(t^{2} / 2\right)} d t
$$

For Quadrature PSK (QPSK), each symbol consists of 2 bits, that is $k=2$. Thus, four signals or message points are produced. These four points on the constellation diagram are equally spaced around a circle (usually spaced at $\pi / 4$ ). Hence, as QPSK's symbol rate is double that of BPSK, it is more spectral efficient than BPSK. The probability of bit error for QPSK is widely regarded as same as that of BPSK.
For Quadrature amplitude modulation (QAM), two digital bit streams are transmitted by modulating the amplitudes of the two carrier waves. The bit error probability, $P_{M Q A M}$, for M-ary QAM scheme is given by:

$$
P_{M Q A M} \cong 2\left(\frac{M}{M-1}\right) Q\left[\sqrt{\frac{6 \lambda}{M^{2}-1}}\right]
$$

For large value of $M$, equation (5) can be written as:

$$
P_{M Q A M} \cong 2 Q\left[\sqrt{\frac{6 \lambda}{M^{2}}}\right]
$$

\subsection{Channel Models in OFDM}

(a) Additive White Gaussian Noise is a basic noise model used to realise the effects of the many random processes that occur in a wireless channel. These random processes can come from some natural sources such as thermal noise and sun. The model does not account for fading, frequency selectivity, interference, non-linearity or dispersion $[17,18]$. Instead, it provides simple models, which give a useful insight into the underlying behaviour of a system before the aforementioned factors, are considered. Therefore, the model can be said to be useful in simulating the background noise of a channel. This model inputs a white Gaussian noise to the signal that passes through it for analysis purposes. For a transmitted signal, $s(t)$, the received signal $r(t)$, is expressed as:

$$
r(t)=s(t)+n(t)
$$

where, $n(t)$ is the additive white Gaussian noise.

The probability density function of the Gaussian noise is expressed thus [8]:

$$
p(z)=\frac{1}{\sigma \sqrt{2 \pi}} e^{-\frac{(z-\mu)^{2}}{2 \sigma^{2}}}
$$

where $\mu$ is the mean and $\sigma$ is the standard deviation (b) Another source of signal degradation in wireless communication is fading, which can be said to be the variation of signal amplitude and phase over frequency and time. Depending on the transmission path, there can either be a dominant line-of-sight (LOS) between the transmitter and the receiver or not. Most times, the 
transmitter and receiver are so far apart that a direct LOS is not possible. Thus, signals utilise the multipaths. In addition, when this happens, applying Central Limit Theorem, each path can be modelled as circularly complex Gaussian random variable with time as the variable. This model is known as Rayleigh fading channel. As there is no dominant LOS, the Gaussian process here is modelled with a zero mean. This model is reasonable for an environment where there is a large number of reflectors and obstacles.

The received signal, $r(t)$, in Rayleigh fading channel can thus be represented as [4]:

$$
r(t)=s(t) \times h(t)+n(t)
$$

where, $n(t)$ is the AWGN with zero mean and unit variance, $h(t)$ is the the random channel matrix having Rayleigh distribution, $s(t)$ is the transmitted signal. The Rayleigh distribution is fundamentally the magnitude, $z$, of the sum of two equal independent orthogonal Gaussian random variables and would have a probability density function $[4,15]$ :

$$
p(z)=\frac{z}{\sigma^{2}} e^{-\frac{z^{2}}{2 \sigma^{2}}}, \quad z \geq 0
$$

\section{METHODOLOGY}

\subsection{Simulation Model}

The proposed OFDM system is simulated using SIMUINK in MATLAB. The performance results for the system using different modulation schemes are obtained using the OFDM parameters listed in Table 1.

Table 1: Parameters for the simulation of the OFDM

\begin{tabular}{ll} 
& model \\
\hline System Parameters & Values \\
\hline System & OFDM \\
Modulation techniques & BPSK/QPSK/16QAM/64QA \\
Fading channel type & M/256QAM \\
Tx and Rx Antenna & SISO \\
FFT/IFFT Points & 256, 512 and 1024 \\
Guard type & Cyclic prefix \\
Cyclic Prefix length & $25 \%$ \\
Doppler shift & 10 Hertz \\
\hline
\end{tabular}

\subsection{Simulation Steps}

1. The system model was put together in Simulink with the constituting blocks described in section 2 .

2. Firstly, the BPSK modulation/demodulation block was put in place whilst implementing only the AWGN channel. The Eb/No of the AWGN channel block parameter was varied from $0-16$ and the simulation was run for a few minutes.

3. The BER is read from the BER calculator block.
4. Steps 2 and 3 are repeated for the following modulation/demodulation block pairs: QPSK, 16 QAM, 64 QAM and 256 QAM.

5. Steps 2,3 and 4 are repeated while varying the number of subcarriers through the values: 256, 512 and 1024.

6. Steps 2, 3, 4 and 5 are repeated, this time using the Rayleigh Fading channel alongside the AWGN channel.

\section{RESULTS AND DISCUSSION}

\subsection{BER Performance Over AWGN At Different Subcarriers}

Here, the BER performance of different modulation techniques over AWGN channel in a SISO-OFDM configuration are presented. The results are presented graphically which shows the BER as a function of the $\mathrm{Eb} /$ No. The modulation techniques considered are BPSK, QPSK, 16 QAM, 64 QAM and 256 QAM. The BER against Eb/No for OFDM with different sub-carriers scenarios under AWGN channel are as shown in Figures 4-6.

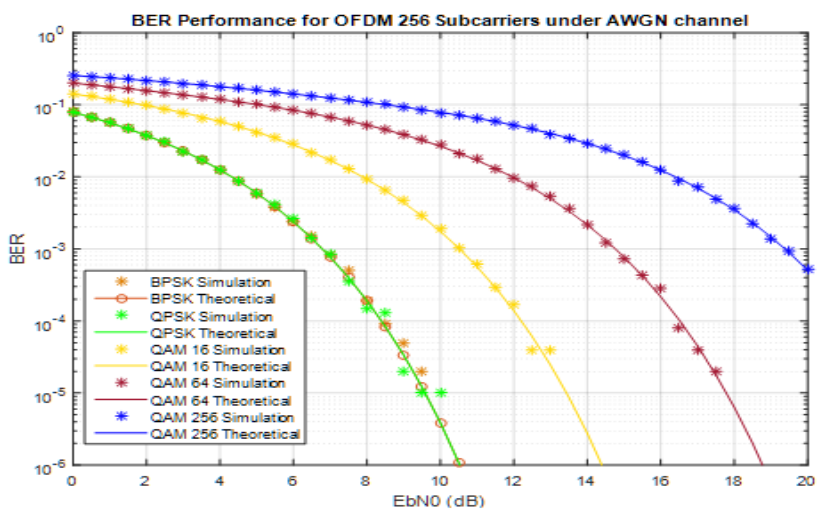

Figure 4: BER against Eb/No for OFDM 256 subcarriers under AWGN channel

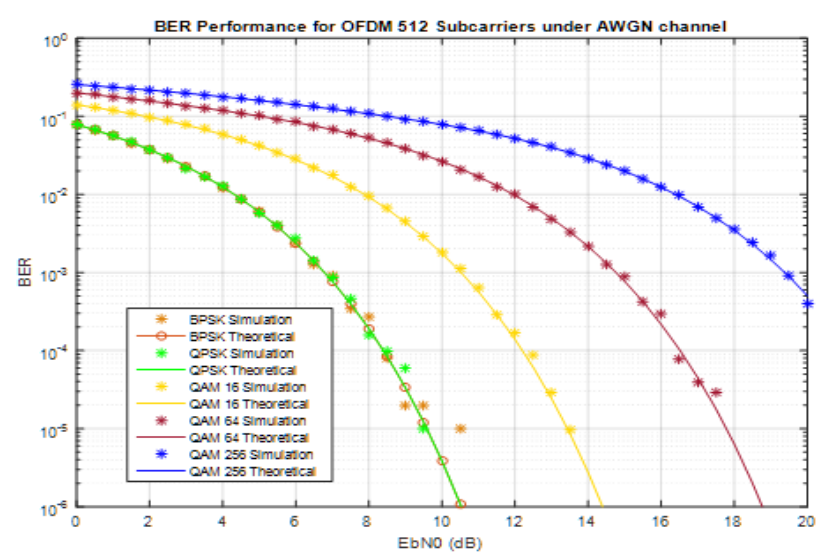

Figure 5: BER against Eb/No for OFDM 512 subcarriers under AWGN channel 


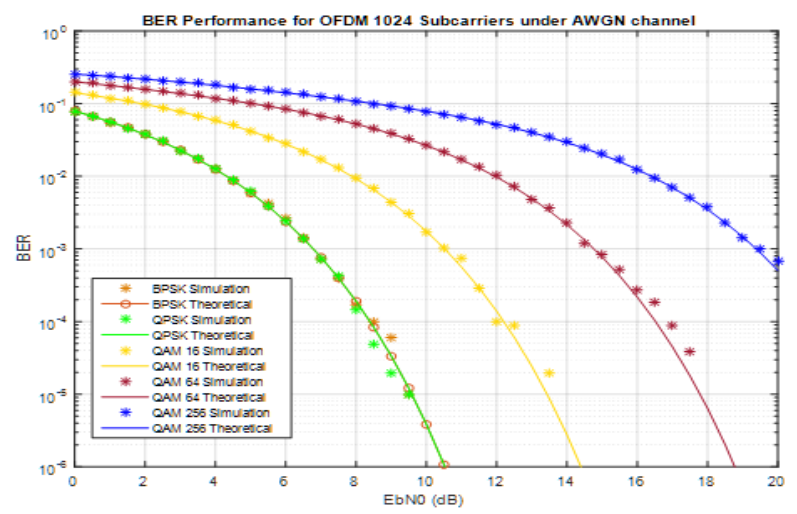

Figure 6: BER against Eb/No for OFDM 1024 subcarriers under $A W G N$ channel

\subsection{BER Performance Over Rayleigh at Different Subcarriers}

Here, the BER performance of different modulation techniques over Rayleigh channel in a SISO-OFDM configuration is presented. The performance was also observed at different FFT points to determine if the number of subcarriers has an effect on the system performance. The same modulation techniques are considered. The BER against Eb/No for OFDM with different sub-carriers scenarios under Rayleigh fading channel are as shown in Figures 7-9.

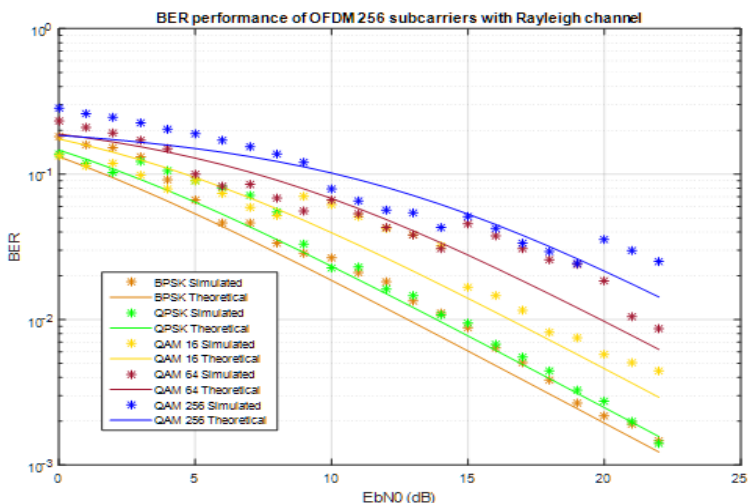

Figure 7: BER against Eb/No for OFDM 256 subcarriers under Rayleigh fading channel

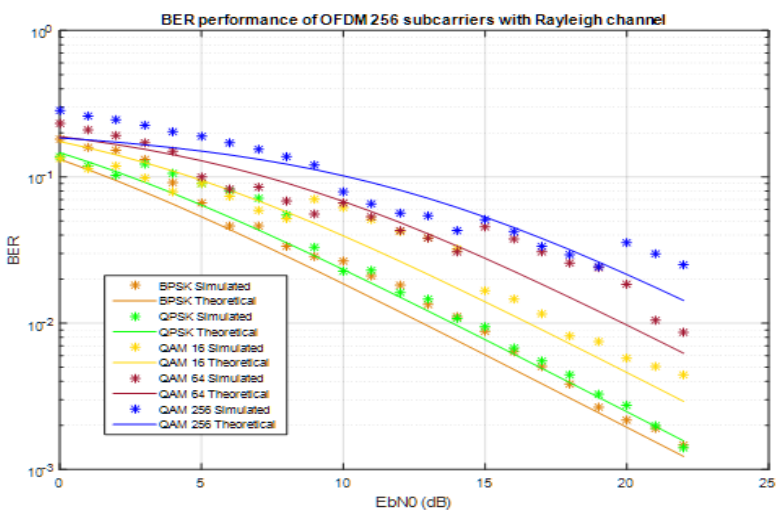

Figure 8: BER against Eb/No for OFDM 512 subcarriers under Rayleigh fading channel

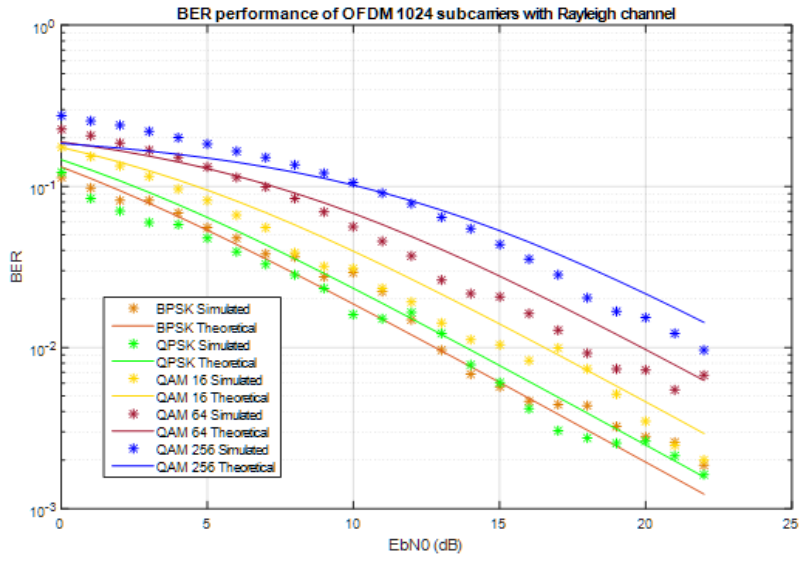

Figure 9: BER against Eb/No for OFDM 1024 subcarriers under Rayleigh fading channel

\subsection{Discussion}

It is seen that the system performs better as the Eb/No increases across all modulation techniques over the AWGN channel. It can also be seen that increasing the number of FFT points does not really affect the performance as they showed similar patterns. For example, 16QAM achieves an Eb/No of 8dB over AWGN when FFT point of 256 and 1024 are employed. It is also observed that the lower order modulation techniques perform better than the higher order counterparts. BPSK and QPSK have similar performance while 16QAM performs better than both 64 QAM and 256 QAM. At FFT point of 1024, 16 QAM achieves a BER of 0.001 at an Eb/No of around $10 \mathrm{~dB}$ while it takes 64 QAM around $14 \mathrm{~dB}$ and 256 QAM around $19 \mathrm{~dB}$ to achieve the same level of BER. Similar patterns are obtained at other FFT points whilst employing a $25 \%$ of the FFT points as virtual carriers in each case.

From Figures 7-9, it is seen that the performance has a similar pattern with AWGN in that the better BER are achieved at higher Eb/No. Nevertheless, it is very clear that the system over AWGN outperforms the system over Rayleigh as none of the modulation techniques achieved a BER of 0.001 . It is observed that lower order modulation still perform better than higher order modulation techniques. 16QAM gives a clearly better performance than 64QAM and 256 QAM. At a subcarrier of 256, a BER of 0.01 for QPSK in the Rayleigh fading channel was achieved at around $18 \mathrm{~dB}$ but at a subcarrier of 1024, same BER was achieved at around $17 \mathrm{~dB}$, a gain of $1 \mathrm{~dB}$. In addition, at a subcarrier of 256, a BER of 0.01 for 64 QAM in the Rayleigh fading channel was achieved at around $21 \mathrm{~dB}$ but at a subcarrier of 1024, same BER was achieved at around $20 \mathrm{~dB}$, a gain of $1 \mathrm{~dB}$. These results compare favourably with related works on the performance of different modulation schemes; in PAPR reduction in OFDM

Vol. 37, No. 3, July, 2018 
signals [19], under sweep jamming [20], and in aeronautical channels [21].

Generally, it can be concluded that increasing the number of FFT points in the OFDM system does not really have a significant effect on the performance of the system as no modulation technique showed a better performance over another. However, this could invariably affect the energy per bit of the signal.

\section{CONCLUSION}

The performance of OFDM system has been evaluated over two channels: AWGN and Rayleigh fading channels, using various modulation schemes; BPSK, QPSK, 16 QAM, 64 QAM and 256 QAM. In addition, the study investigated whether the number of FFT points employed has an effect on the system performance. The system performance was compared for all the employed modulation techniques, over both channels. Ultimately, similar comparison was made for the system when transmitting at various FFT points. Generally, it can be concluded that over both AWGN and Rayleigh fading channels, lower order modulation schemes perform better than the higher order schemes but this comes at the expense of the data rate, as lower order schemes have lower data rates than their higher order counterparts. In addition, all the modulation schemes performs better in AWGN channel than Rayleigh fading channel. When transmitting at higher subcarriers, no improvement is observed in any of the modulation schemes. Therefore, transmitting at high subcarriers generally does not have an effect on OFDM transmission although, transmit power could be affected. Our future work will focus on the hardware implementation of the proposed MIMO-OFDM system.

\section{REFERENCES}

[1] Sharma, D. and Srivastava, P. OFDM Simulator Using MATLAB. International Journal of Emerging Technology and Advanced Engineering, 3(9), 493496, 2013.

[2] Gupta, R., and Parida, S. Challenges and Opportunities: Mobile Broadband. International Journal of Future Computer and Communication, 2(6), pp. 660-664, 2013.

[3] John, S. N., Akinola, E., Ibikunle, F., Ndujiuba, C. U., and Akinaade, B. Modeling of orthogonal frequency division multiplexing (OFDM) for transmission in broadband wireless communications. Journal of Emerging Trends in Computing and Information Sciences, 3(4), 534539, 2012.
[4] Kansal, L., Kansal, A. and Singh, K.. Performance analysis of MIMO-OFDM system using QOSTBC code structure for M-QAM. Canadian Journal on Signal Processing, 2(2), 4-15, 2011.

[5] Rohling, H. (Ed.). OFDM: concepts for future communication systems. Springer Science \& Business Media, 2011.

[6] Ghosh, S.. Performance evaluation on the basis of Bit error rate for different order of Modulation and different length of Sub channels in ofdm system. arXiv preprint arXiv:1406.7405, 2014.

[7] Kamboj, A. and Kaushik, G. Study \& Simulation of OFDM System. International Journal of Modern Engineering Research (IJMER), 2(1), pp. 235-241, 2012.

[8] Katariya, A., Yadav, A. and Jain, N. Performance elevation criteria for OFDM under AWGN fading channel using IEEE 802.11a. International Journal of Soft Computing and Engineering (IJSCE), 1(3), pp. 10-13, 2011.

[9] Bodhe, R., Narkhede, S. and Joshi, S.. Design of Simulink Model for OFDM and Comparison of FFTOFDM and DWT-OFDM. International Journal of Engineering Science and Technology, 4(5), 19141924, 2012.

[10] Mohamed, M. A., Samarah, A. S. and Allah, M. F. Study of performance parameters effects on OFDM systems. International Journal of Computer Science Issues, 9(3), 1694-0814, 2012.

[11] Dixit Dutt Bohra, A. B. Bit Error Rate Analysis in Simulation of Digital Communication Systems with Different Modulation Schemes. International Journal of Innovative Science, Engineering \& Technology, 1(3), pp. 406-413, 2014.

[12] Shashikant, S., and Dhawan, D.. Cyclic prefix optimization of OFDM system. IOSR J. Electron. Commun. Eng., 9(3), 79-82, 2014.

[13] Cho, Y. S., Kim, J., Yang, W. Y. and Kang, C. G.. MIMO-OFDM wireless communications with MATLAB. John Wiley \& Sons, Singapore, 2010.

[14] Ahmed, E., Aziz, W., Saleem, S., and Islam, Q. Performance Analysis of OFDM System for Different Channel Lengths and Multipath Channel Taps. Advances in Electrical Engineering Systems, 1(2), 124-128, 2012.

[15] Achra, N., Mathur, G. and Yadav, R. P. Performance analysis of MIMO OFDM system for different modulation schemes under various fading channels. International Journal of Advanced Research in Computer and Communication Engineering, 2(5), 2098-2103, 2013.

[16] Panicker, N. V. and Sukesh, A. K. BER Performance Evaluation of Different Digital Modulation Schemes for Biomedical Signal Transceivers under 
Bit Error Rate Analysis of Different Digital Modulation Schemes in Orthogonal Frequency Division ...S. O. Ajose, et al.

AWGN and Fading Channel

Conditions. International Journal of Engineering and Advanced Technology, 3(5), pp 212-215, 2014.

[17] Gupta, S. and Sharma, H. Performance Investigation for different modulation techniques in WCDMA with multipath fading channels. International Journal of Advanced Research in Computer Science and Software Engineering, 2(7), 20-23, 2012.

[18] Anioke, C. L., Nnamani, C. O. and Ani, C. I. Review of Wireless MIMO Channel Models. Nigerian Journal of Technology, 35(2), 381-391, 2016.

[19] Mowla, M., Paul, L. C., \& Hasan, M. Comparative Performance Analysis of Different Modulation
Techniques for PAPR Reduction of OFDM Signal. arXiv preprint arXiv:1406.3019, 2014.

[20] Choi, D. Y., Kim, W. K., Kim, J. H., and Cho, H. Performance of analog and digital modulation schemes under sweep jamming. In IEEE 8th International Conference on Ubiquitous and Future Networks, Vienna, Austria, pp.13-15, 5-8 July, 2016. DOI: 10.1109/ICUFN.2016.7536970.

[21] Neeraja, C. J., and Nandan, S. Performance comparison of different modulation schemes in aeronautical channels. In IEEE International Conference on Advanced Communication, Control and Computing Technologies, Ramanathapuram, India, pp. 702-706, 8-10 May, 2014. DOI: 10.1109/ICACCCT.2014.7019181. 